\title{
TRANSCRIÇÃO E RECONSTRUÇÃO DIGITAL - FERRAMENTAS NA ANÁLISE DE ARQUITETURA
}

\author{
CLARO, Marcel Alessandro
}

UFU - Universidade Federal de Uberlândia, e-mail: marcelclaroarquiteto@gmail.com

RIBEIRO, Patrícia Pimenta Azevedo

UFU - Universidade Federal de Uberlândia, e-mail: pparibeiro2003@yahoo.com.br

\begin{abstract}
RESUMO
Este artigo decorre de um capítulo da Dissertação de Mestrado defendida em 2017 e demonstra a importância da Transcrição e Reconstrução digital como ferramenta para análise, compreensão e visualização do espaço arquitetônico projetado e não construído. Nos últimos anos a realidade virtual e a computação gráfica revolucionaram o mundo em todas as suas instâncias, inclusive na arquitetura e urbanismo. Está presente nos processos de concepção, projetação, produção e representação. Nesta pesquisa adotou-se como metodologia uma abordagem prática e um estudo de caso - Hotel Tropical de Manaus - projeto do arquiteto Sergio Bernardes, não construído. Afim de gerar linhas de investigação foi fundamental a revisão de fontes primárias de arquivos sobre o arquiteto e o projeto. Depois de recolhidas todas as provas documentais, a aplicação do método da Transcrição digital - gerados em plataformas digitais (CAD e BIM) - permitiu a Reconstrução Digital feita em técnicas de modelagem 3D mais adequadas para responder às perguntas específicas do pesquisador sobre o projeto transcrito. Essa metodologia possibilitou criar uma nova iconografia, um espaço virtual que admite explorações, sensações, experiências e visualizações do projeto no intuito de enriquecer os trabalhos acadêmicos e científicos sobre projetos de arquitetura não construídos.
\end{abstract}

Palavras-chave: Transcrição e Reconstrução Digital, Edifícios não construídos, Modelagem 3D, Análise de Projeto.

\begin{abstract}
This article stems from a chapter of the Master Dissertation defended in 2017 and demonstrates the importance of Digital Transcription and Reconstruction as a tool for the analysis, understanding and visualization of the designed and not constructed architectural space. In recent years virtual reality and computer graphics have revolutionized the world in all its instances, including architecture and urbanism. It is present in the processes of conception, design, production and representation. In this research, a practical approach and a case study Tropical Hotel of Manaus - project of the architect Sergio Bernardes, not built, were adopted as methodology. In order to generate research lines it was fundamental to review primary sources of archives about the architect and the project. After collecting all the documentary evidence, the application of the Digital Transcription method - generated in digital platforms (CAD and BIM) allowed the Digital Reconstruction made in 3D modeling techniques more adequate to answer the specific questions of the researcher about the transcribed project. This methodology allowed to create a new iconography, a virtual space that admits explorations, sensations, experiences and visualizations of the project in order to enrich the academic and scientific works on architecture projects not constructed.
\end{abstract}

Keywords: Transcription and Reconstruction Digital. Buildings not built, 3D Modeling, Project Analysis.

CLARO, M. A.; RIBEIRO, P. P. A. Transcrição e reconstrução digital - ferramentas na análise de arquitetura. In: SIMPÓSIO BRASILEIRO DE QUALIDADE DO PROJETO NO AMBIENTE CONSTRUÍDO, 6., 2019, Uberlândia. Anais... Uberlândia: PPGAU/FAUeD/UFU, 2019. p. 812-820. DOI https://doi.org/10.14393/sbqp19075. 


\section{INTRODUÇÃO}

Este artigo objetiva demonstrar como a revolução tecnológica criou novas ferramentas de análise arquitetônica, permitindo uma melhor visualização e compreensão do espaço projetado mas não construído. Apresenta a experiência prática da transcrição e reconstrução digital do Hotel Tropical de Manaus, um projeto arquitetônico (1970), não construído, do arquiteto Sergio Bernardes.

Nos últimos trinta anos - final do século XX e início do XXI - a realidade virtual e computação gráfica revolucionaram o mundo em todas as suas instâncias, inclusive na arquitetura e urbanismo. Seus reflexos estão presentes nos processos de concepção, projetação, produção e representação, dos projetos de arquitetura e urbanismo, em modelagens tridimensionais, renderizações, modelagens paramétricas, fabricações digitais e simulações.

A Realidade Virtual (RV) é um conjunto de tecnologias que permite a um utilizador interagir com ambientes ou objetos virtuais, simulações de espaços existentes ou imaginários, através de um computador ou uma interface digital em tempo real.

Para Stever (1992), o conceito de presença é a sensação de estar presente em um ambiente mediado por processos mentais. Quando estes processos são auxiliados por tecnologias de informação e comunicação têm-se a "telepresença". Consequentemente toda e qualquer experiência de telepresença é uma realidade virtual.

A experienciação de espaços através de realidade virtual está diretamente ligada à imersão que este ambiente proporciona. Os modelos tridimensionais virtuais projetados em telas de computadores ou televisões continuam como uma experiência bidimensional, pois não é possível perceber as profundidades do espaço tridimensional. Para uma imersão mais profunda, é preciso o uso de tecnologias e aparatos óticos para construção de uma imagem estereoscópica que simule a visão e permita a sensação de profundidade enquanto se vivencia o espaço tridimensional virtual.

Podemos ter experimentações espaciais no meio digital utilizando técnicas diferentes. Uma das formas de experimentações espaciais, são as realizadas em CAVE.

CAVE é uma pequena sala onde são projetadas cenas geradas por computador, gráficos e imagens em três dimensões em todas as paredes, teto e chão, que podem ser visualizados, navegados e manipulados pelos usuários através de dispositivos (óculos, capacetes, luvas) na interação e exploração do espaço digital, objetos e pessoas num mundo virtual. Pode-se produzir todos os tipos de edifícios, espaços e objetos com texturas e luzes que simulem e que tragam o usuário a uma proximidade do "mundo real" para que a experiência seja mais próxima a tudo aquilo que ele vivencia no seu cotidiano.

Modelagem tridimensional virtual ou Modelo 3D é uma representação matemática de uma superfície tridimensional, forma, objeto, personagem ou cenário por um software 3D que produz uma malha geométrica complexa, polígonos, vértices para produzir imagens virtuais destes elementos.

As modelagens tridimensionais virtuais podem ser feitas com vários softwares disponíveis no mercado, alguns com licenças gratuitas e outros pagos. Entre 
eles temos o AutoCAD, Sketchup, Blender, Rhinoceros, Grasshopper, Revit, ArchiCAD e muitos outros. Os mais usados para modelagem em arquitetura são o Sketchup (CAD), o Revit e o ArchiCAD estes dois últimos possuem tecnologia BIM.

O desenho assistido por computador (CAD - Computer Aided Design) tem sua estrutura de dados baseada em entidades geométricas genéricas e para quaisquer atualizações do modelo são necessários novos desenhos dos objetos.

O BIM - Building Information Modeling ou Informação do Modelo de Construção, é um conceito metodológico do processo representativo e projetual onde estão inseridas as informações necessárias para sua construção - projeto arquitetônico, projeto estrutural, projetos complementares, materiais, acabamentos, custos. Os sistemas BIM possuem elementos paramétricos (parâmetros conectados entre si) onde as alterações são atualizadas de forma instantânea no projeto. Os softwares com a tecnologia BIM permitem a simulação da construção do projeto no meio digital e a inserção de informações em cada etapa de projeto, da fundação ao acabamento, gerar planilhas, custos, plantas, cortes, perspectivas, detalhes e estruturas. Essas informações são inseridas em um banco de dados que faz uma atualização constante em todo o processo de projeto. Essa tecnologia traz consigo um novo sistema de trabalho, o sistema colaborativo, onde cada profissional que participa do processo, pode modificar o projeto e têm seus arquivos atualizados em tempo real.

A ampliação do uso das ferramentas digitais aumentaram a capacidade de inserção de informações nos modelos tridimensionais, cada vez mais complexos e poderosos na produção de simulações. Por isso, tornaram-se ferramentas fundamentais para as análises de projeto no âmbito da pesquisa.

Muitas obras de arquitetura e urbanismo permanecem não-construídas ou nunca serão, talvez por conterem ideias à frente de seu tempo, tecnologias que ainda não poderiam ser empregadas, custos, experimentações e ideais que refletiam um imaginário de futuro de uma sociedade, ou ainda, utópicas e visionárias. Porém, quase todos estes projetos não-construídos contêm documentos gerados pelo arquiteto-autor que podem trazer novas visões sobre os espaços imaginados.

O uso da modelagem digital para reconstruir a arquitetura perdida (destruída ou demolida) ou não edificada é um processo bem-sucedido que contribui para aumentar a nossa compreensão espacial desses projetos (NOVITSKI, 1998; FORTE; SILIOTI, 1997). Outros autores vêm pesquisando técnicas digitais para recuperação da memória de edifícios históricos e patrimônio arquitetônico. De acordo com Sky e Stone (1983) e Stamp (2007) tais técnicas digitais são utilizadas na reinterpretação e análises de documentos, imagens e fotos sobreviventes da ação do tempo. Novitski (1998) e Burry (2008) mostram que esse processo pode trazer novas informações dos edifícios perdidos ou não edificados.

A informação gerada em 3D pode ser considerada como o núcleo do processo de conhecimento, porque cria uma reflexão, um retorno entre o interativo (usuário), o meio digital e o pesquisador onde a Realidade Virtual é capaz de gerar esses processos de conhecimento e conexão entre eles. 
Em um ambiente imersivo de uma possível arqueologia virtual, proposta pela utilização de modelos tridimensionais de arquitetura não construída, as informações dependem de relações de sua própria natureza (desenhos originais do projeto) e da não neutralidade de como é percebida, produzida e processada pelo pesquisador. Esse "tempo passado" é codificado por um processo de simulação e as técnicas e tecnologias que são usadas nesse processo influenciam de maneira decisiva todas as interpretações. A perda de informações, quando escolhas são equivocadas, afetam as compreensões e análises.

Essa arqueologia virtual fornece ferramentas adequadas para a compreensão do modelo e qual tipo de simulação será produzida para que a interação entre o usuário e o modelo gerado se torne um processo inovador de aprendizagem, onde o observador (pesquisador) seja capaz de interpretar e analisar as possíveis informações que não foram imaginadas ou previstas pelo arquiteto autor do projeto.

\section{TRANSCRIÇÃO DIGITAL}

O termo transcrição é associado à ideia da transcrição de áudios em textos e vice-versa. A veracidade dos elementos analisados, palavras, textos e ideias, é de suma importância para uma perfeita transcrição e preservação do autor. Faz-se então, uma analogia ao termo transcrição digital para o método proposto neste trabalho.

Toma-se como Transcrição Digital todo o processo de desenho da documentação dos projetos de arquitetura e urbanismo coletadas (desenhos originais das plantas, cortes, elevações, fotos de maquetes físicas, croquis e perspectivas) em plataformas digitais (softwares CAD e BIM) para a posterior "reconstrução" do projeto em modelagens tridimensionais digitais. Esse processo exige habilidades semelhantes às instituídas para a restauração de obras de arte onde a preservação do caráter original é fundamental para a interpretação da obra. No entanto, a quantidade e veracidade das informações contidas no banco de dados produzido durante a pesquisa, trazem variáveis que devem ser verificadas e comprovadas para que a Transcrição Digital do projeto seja a mais próxima das intenções e conceitos adotados pelo arquiteto estudado. Esta pesquisa utilizou principalmente dados sobre o projeto do Hotel Tropical de Manaus contidos no NPD- UFRJ.

A Transcrição Digital dos documentos de projeto deve ser cuidadosa. 0 processo de Transcrição Digital se inicia após uma seleção prévia dos projetos que serão transcritos pela pesquisa documental. É preciso que toda a documentação do projeto a ser transcrito já tenha sido selecionada e organizada. A seleção dos documentos irá proporcionar percursos de análises, trilhar caminhos de pesquisa e questões relevantes para encaminhar a investigação dos projetos.

Também é importante que sejam os documentos originais do projeto, pois documentos que já foram modificados podem ter sofrido interferências e interpretações que não são do autor do projeto.

Pode-se ter documentos originais de um mesmo projeto, mas com datas diferentes. Para tanto busca-se fazer uma organização cronológica e sobrepor 
os modelos para verificação das ideias e soluções que o arquiteto fez sobre o projeto em diferentes tempos.

A qualidade das informações em cada projeto a ser transcrito influenciará diretamente no processo de Transcrição Digital, pois quanto mais precisas as informações menos lacunas e inconsistências. Esse fato diminui possíveis interferências e interpretações do analista que possam desconfigurar ou transformar o projeto de maneira a perder os conceitos e sua essência.

Podemos ter a falta de documentação precisa dos projetos analisados. Para atenuar essa situação torna-se fundamental uma pesquisa paralela sobre o arquiteto e sua atuação profissional a fim de encontrar em seus outros projetos informações relevantes sobre o autor e seu processo de projeto. A pesquisa de projetos datados do mesmo período do projeto que está em análise, projetos com semelhanças significativas como sistemas construtivos e soluções espaciais, geometrias recorrentes, coberturas, aberturas, padrões estruturais, materiais, livros e tratados escritos pelo arquiteto, e quaisquer outros documentos que possam trazer informações relevantes sobre o projeto em análise como publicações, cartas, jornais, fotografias.

No entanto, a quantidade e veracidade das informações contidas no banco de dados trarão muitas variáveis que devem ser verificadas e comprovadas para que a Transcrição Digital do projeto seja a mais próxima possível das intenções e conceitos adotados pelo arquiteto estudado.

Assim, cabe ao pesquisador fornecer subsídios suficientes para validar o modelo e as relações de análise que se busca através dele. Além de preservar o arquiteto, deve-se manter a integridade dos conceitos que o arquiteto usou no projeto e explorar o conteúdo emocional, metafórico e simbólico da sua obra.

A etapa seguinte consiste em transcrever toda a documentação coletada em documentos digitais produzidos em softwares de desenho assistido por computador (CAD). Para isso utiliza-se da digitalização dos documentos originais que são inseridos e importados para os softwares de desenho. Após inseridos os desenhos nos softwares inicia o processo de desenho digital.

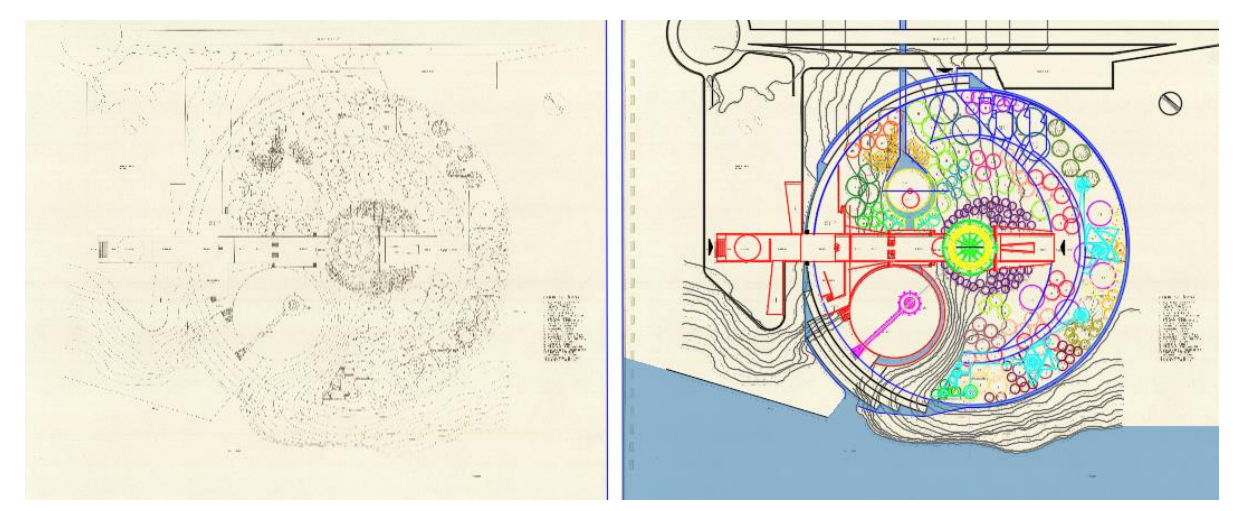

Figura 1 - Transcrição Digital dos desenhos originais do projeto do Hotel Tropical de Manaus 1970 (Sergio Bernardes) Fonte: Autor (2017)

Salienta-se que esse método proposto se enquadra em pesquisas de projetos que foram produzidos de forma analógica, ou seja, desenhados à mão. Os 
projetos já produzidos em meios digitais poderão ser reconstruídos digitalmente sem o processo da Transcrição Digital.

As imagens originais do projeto depois de escaneadas e inseridas num software de tratamento de imagens são exportadas para o programa CAD e usadas como bases para os desenhos iniciais do processo de Transcrição e Reconstrução Digital.

\section{RECONSTRUÇÃO DIGITAL}

Reconstrução Digital é o processo de modelagem digital (modelos 3D) para a produção de novos desenhos e documentos do projeto estudado em modelos tridimensionais virtuais que permitam as simulações e vivências espaciais. Todos os documentos gerados no processo de Transcrição Digital são utilizados para a Reconstrução Digital.

Os arquivos criados em software CAD são importados para os programas de modelagem 3D (softwares Sketchup e Revit). A organização dos desenhos segue as mesmas indicações como estão nas plantas e arquivos originais do projeto e também às suas localizações e dimensões.

Podemos ter a Reconstrução Digital tanto de projetos construídos como a de projetos não-construídos.

Os projetos que já foram edificados permitem uma maior quantidade e qualidade de informações disponíveis para Transcrição e Reconstrução Digital pois é possível fazer um levantamento no local e produzir toda a documentação as builtl. $O$ acesso aos projetos de execução e compatibilizações são facilitados pois estão de domínio dos proprietários ou órgãos governamentais, além da possibilidade de visitação e análise direta no local.

Para trabalhar com grande quantidade e variedade de informações, a Reconstrução Digital deve apoiar na tecnologia dos softwares BIM, pois, em qualquer etapa da modelagem, há inserção de informações decorrentes das pesquisas paralelas sobre o projeto e o arquiteto, diretamente no modelo gerado, que é atualizado em tempo real sem a necessidade de uma nova modelagem. As diferentes combinações destes fatores na reconstrução digital, permite distintas formas para sua exploração no meio digital e gerar experiências qualitativas diversas, pois cada espaço virtual reconstruído traz características e percepções únicas.

A Reconstrução Digital visa a não interferência no projeto. Ela anseia trazer novas perspectivas e desenhos do projeto que mostrem outros caminhos para investigações teóricas e projetuais sobre o arquiteto autor do projeto e através das análises dos modelos reconstruídos, refletir diferentes escolas de pensamento, inferências e posições para a ampliação do repertório sobre o arquiteto pesquisado. Por exemplo, intenções em relação à texturas e iluminação que não foram considerados, pois não havia nas pesquisas dos documentos desenhos renderizados à mão e sim um texto ou diagrama com a

\footnotetext{
1 As Built é uma expressão inglesa que significa "como construído". Os projetos As Built consistem no levantamento de todas as dimensões e características pertinentes de uma edificação e/ou de suas instalações, posteriormente transformadas em representações técnicas (plantas, cortes, fachadas e outros).
} 
informação, que a Reconstrução Digital e simulações de iluminação no modelo digital clarifiquem essa intenção de projeto do arquiteto.

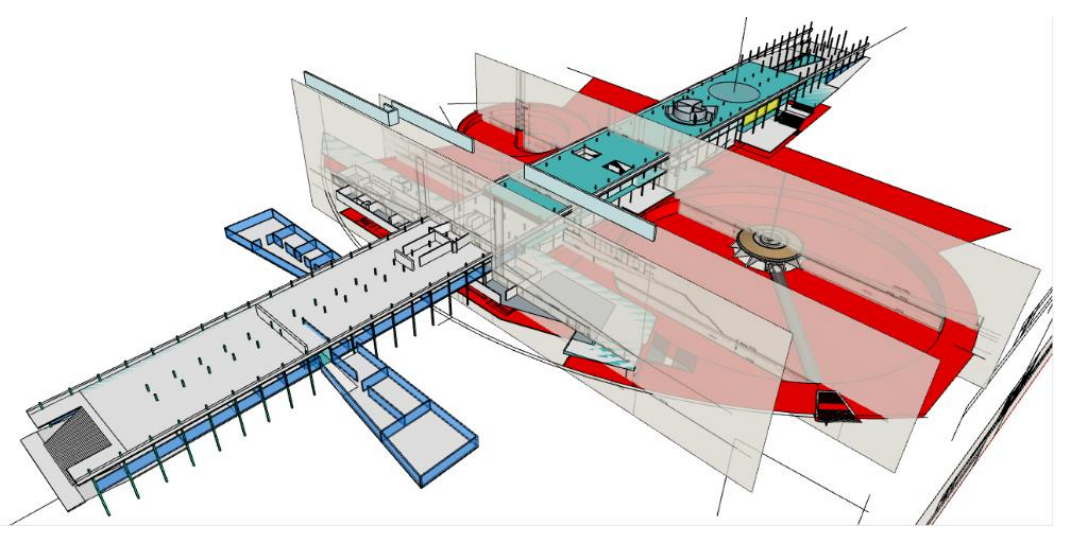

Figura 2 - Modelagem a parir dos planos transcritos -

Fonte: Autor (2017)

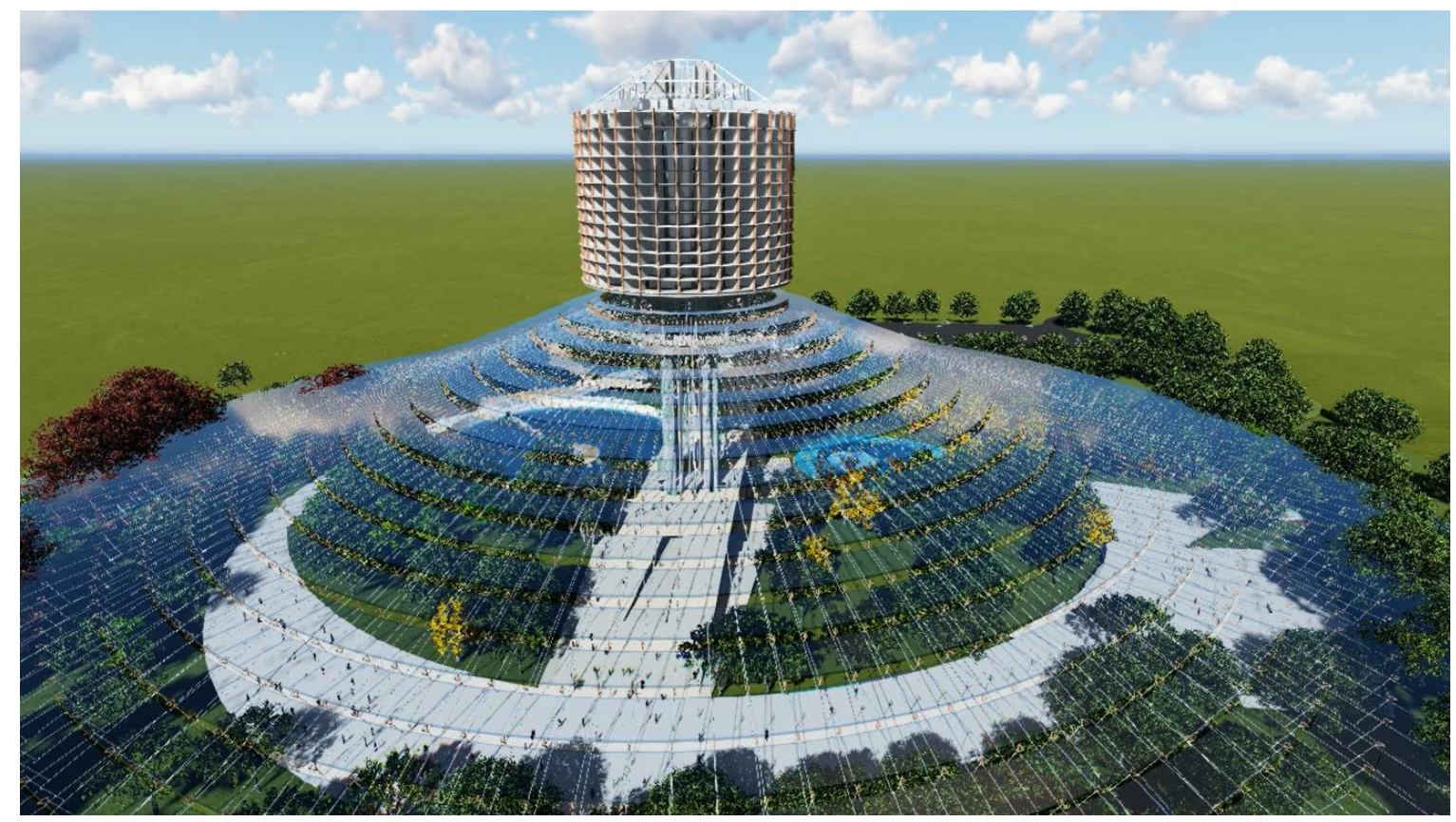

Figura 3 - Modelo 3D finalizado e inserido no software de criação de percursos virtuais Fonte: Autor (2017)

Outras situações podem surgir, soluções estruturais que na época da proposta do projeto eram consideradas impossíveis ou improváveis e compará-las com as técnicas atuais dos sistemas estruturais para verificar as possibilidades de execução do projeto, situações de ensaio de cargas estruturais previstas pelo arquiteto porem não desenhadas

Além da espacialidade, visualização e percursos a Transcrição Digital e a Reconstrução Digital de edifícios não-construídos fornece conceitos, ideias e influências sobre o processo de projeto, permite a comparação das ideias do pesquisador com as análises de outros pesquisadores de um mesmo projeto, no intuito de enriquecer, cada vez mais, os trabalhos acadêmicos e científicos sobre os projetos de arquitetura não-construídos e criar uma nova iconografia, um espaço virtual para explorações, sensações, experiências e visualizações do projeto. Vislumbra-se a oportunidade de vivenciar e experienciar os 
espaços projetados, nunca "visitados", de forma virtual, que podem ser sintetizados em conhecimento e aprendizado sobre a própria arquitetura, discussões latentes tornam-se realidades, e novas ideias surgem.

\section{CONSIDERAÇÕES}

Essa metodologia possibilitou criar uma nova iconografia, um espaço virtual que admite explorações, sensações, experiências e visualizações do projeto no intuito de enriquecer, cada vez mais, os trabalhos acadêmicos e científicos sobre projetos de arquitetura não construídos.

No caso específico deste estudo, a transcrição e reconstrução digital do Hotel Tropical de Manaus permitiu visualizar e analisar o projeto desde a sua implantação no terreno, conexão com o entorno, sua plástica, sua espacialidade, suas belas soluções construtivas dos anéis da torre e da cúpula.

Para uma maior qualidade no processo de transcrição é importante que o desenho original seja respeitado ao máximo em suas dimensões e níveis, pois interferem diretamente na produção do modelo 3D. É sabido que quando digitalizamos imagens de projetos originais feitos à mão sofremos o risco de perdermos uma quantidade de informação que pode acarretar em imprecisões dimensionais. Mas, quando se trata das análises, é possível fazer medidas aproximadas no início do processo e ao longo da modelagem, verificar as medidas que seriam mais corretas a serem usadas e é possível antever algumas tomadas de decisões do arquiteto que fez o projeto, graças ao uso de simetrias, modulações, dimensões básicas e padrões presentes na construção civil, além de redimensionar elementos e utilizar de imagens de texturas e obter medidas aproximadas dos materiais presentes no processo de reconstrução digital.

Portanto, acredita-se que o método aqui apresentado pode gerar um grande ganho para as pesquisas de projetos não-construídos ou demolidos na geração de novos olhares e questionamentos sobre arquitetura e urbanismo, além de enriquecer as pesquisas acadêmicas com a possibilidade obter novos desenhos e imagens que foram gerados pela Transcrição e Reconstrução Digital a partir de fotos, croquis, esquemas e desenhos de projeto.

Ao longo do percurso percebeu-se que um campo em aberto seria aplicar e testar o método da Transcrição e Reconstrução Digital em outros projetos não executados para reafirmar a sua validade nas análises de projetos arquitetônicos não construídos. Propor uma modelagem mais subjetiva dos projetos para verificar se a análise pelos modelos 3D gerados não sofrerá de grandes discrepâncias, sujeitas as subjetividades dos pesquisadores e inseri-la de um modo mais livre e interpretativo para fomentar discussões mais díspares sobre um mesmo projeto arquitetônico ou urbanístico não construído.

\section{REFERÊNCIAS}

BURRY. M. Gaudi Unseen: Completing the Sagrada Familia. JOVIS, Verlag, 2008. FORTE, M.; SILIOTTI, A. Virtual archaeology: re-creating ancient worlds. New York: Harry N. Abrams, 1997. 
NOVITSKI, B. J. Rendering Real and Imagined Buildings: The Art of Computer Modeling from the Palace of Kublai Khan to Le Corbusier's Villas. Gloucester, Massachusetts: Vol. Rockport Publishers, 1998.

SKY, A; STONE, M. Unbuilt America: Forgotten Architecture in the United States from Thomas Jefferson to the Space Age, Abbeville. Press Inc, United States. 1983.

STAMP, G. Britain's lost cities. Londres: Aurum, 2007

STEUER, J. Defining Virtual Reality: Dimensions Determining Telepresence. Journal Communication. Vol. 42, Issue 4. P. 73-93, 1992.

WEBB, N. J. Digital Re-analysis of Lost or Unbuilt Architecture. The Liverpool School of Architecture, University of Liverpool, UK, 2012. 